\title{
LETTER
}

\section{Bedside ultrasound monitoring of prone position induced lung inflation}

\author{
David Rousset ${ }^{1}$, Benjamine Sarton ${ }^{1,2}$, Beatrice Riu' ${ }^{1}$, Benoit Bataille ${ }^{3}$ and Stein Silva ${ }^{1,2^{*}}$ @on behalf of the PLUS \\ study group
}

(c) 2021 The Author(s)

\section{Dear Editor,}

To date, lung computed tomography (CT) scan is the gold standard to assess the distribution of acute respiratory distress syndrome (ARDS) patient's lung strain [1]. Since CT-based methods are impractical and cannot be used to monitor ARDS lung characteristics throughout prone ventilation, methods feasible at the bedside are very attractive and lung ultrasound (LUS) is one of them. We aimed to compare for the first time, LUS to the endexpiratory lung volume (EELV, measured by automated nitrogen washout/washin technique) [2] for assessing prone positioning-induced lung inflation.

At variance with previous reports [3] and because at best we can only expect LUS techniques to quantitate the degree of "inflation" induced by lung recruitment, we have decided to compare LUS to a lung inflation reference method (EELV), rather than using arterial gas exchange (which depends on both lung ventilation and perfusion) as the main study endpoint. A threshold of EELV of $500 \mathrm{ml}$ was used to define a binarized prone positioning response (responders vs not responders) [2]. Ultrasound examinations were performed and analyzed only by physicians with advanced LUS experience and previously reported interobserver agreement [4, 5]. Forty-five patients with moderate-to-severe ARDS $\left(\mathrm{PaO}_{2} / \mathrm{FiO}_{2}<150\right.$ with $\mathrm{FiO}_{2}$ at least 0.6 and positive endexpiratory (PEEP) at least $5 \mathrm{mmHg}$ ) were prospectively included. LUS data, arterial blood gas analysis, and ventilator variables were systematically recorded immediately before prone positioning (PP), $1 \mathrm{~h}$ (early) and $16 \mathrm{~h}$ (late) after PP onset. Lung hyperinflation was not specifically

\footnotetext{
*Correspondence: silva.s@chu-toulouse.fr

${ }^{1}$ Critical Care Unit, University Hospital of Purpan, 31300 Toulouse, France Full author information is available at the end of the article David Rousset and Benjamine Sarton have contributed equally. Members of the PLUS study group are listed in the Acknowledgement section.
}

assessed. Thereby, we have observed in this proof-of-concept study that:

- LUS score variations throughout PP session (Fig. 1, upper panel), but not basal LUS data in a supine position, are significantly associated with PP response both in terms of lung inflation and blood oxygen level improvement.

- PP-induced lung inflation greater than $500 \mathrm{ml}$ can accurately be estimated by a LUS reaeration score [3] of 10 or greater (Fig. 1, upper panel).

- LUS appears to be a reliable bedside tool to evaluate regional PP-induced gain or loss of lung aeration (Fig. 1, lower panel). Moreover, ultrasound analysis of pulmonary aeration changes at both early and late PP assessments, showed that a significant response to PP was related to a reduced number of pattern's transitions: B2 (multiple coalescent B lines) to normal, C (lung consolidation) to normal and $\mathrm{C}$ to B1 (multiple well-defined either regularly spaced 7-mm apart or irregularly spaced B lines). It is worth noting, that at the late-PP time point a significant increase of B-lines profiles was also observed in previously normally aerated lung regions.

- Repeated LUS assessment across the time, accurately allow lung inflation monitoring during PP sessions (Fig. 1, upper and lower panels). However, neither LUS nor EELV can detect lung hyperinflation and as a consequence, should not be used in isolation as methods of respiratory monitoring in ARDS.

We believe that our data make a significant contribution to advancing the understanding and care of ARDS. We suggest that these findings have clinical implications and might be particularly relevant for further personalize the use of PP in this challenging clinical setting. 


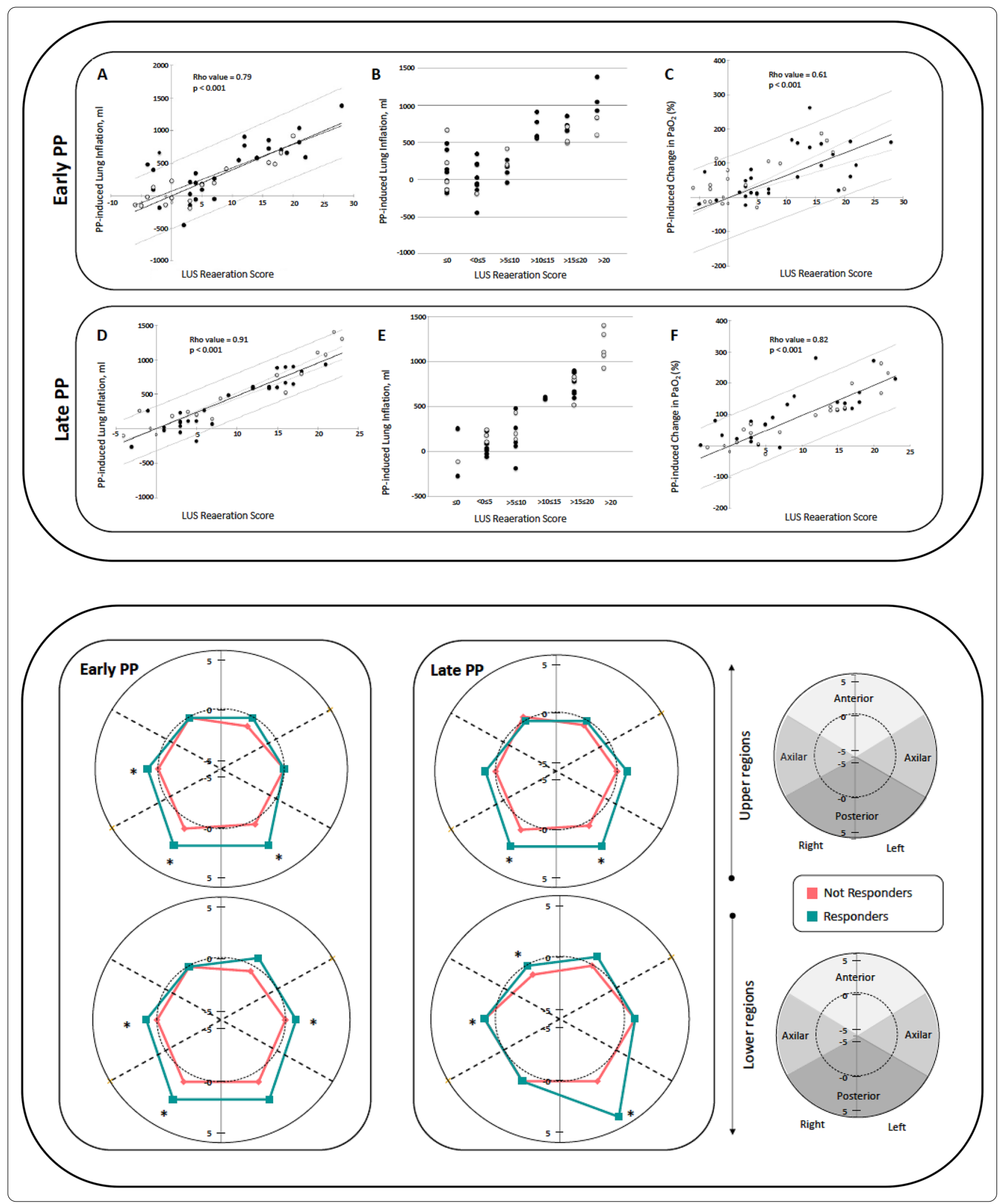


(See figure on previous page.)

Fig. 1 Correlation between ultrasound reaeration score and lung inflation induced by early $(\mathbf{A})$ and late (D) prone positioning (upper panel). Lung inflation was measured as end-expiratory lung volume (EELV) using the multibreath nitrogen-wash-out technique. Accuracy of lung ultrasound score for estimating early $(\mathbf{B})$ and late $(\mathbf{E})$ prone positioning induced lung inflation. Correlation between ultrasound reaeration score and early $(\mathbf{C})$ and late $(\mathbf{F})$ prone positioning induced increase in $\mathrm{PaO}_{2}$. Early and late time points correspond to study assessments made at $1 \mathrm{~h}$ and $16 \mathrm{~h}$ after prone positioning onset, respectively. Each closed circle represents an individual patient. Gray lines represent 95\% confidence intervals. Black circles represent patients with diffuse loss of aeration; open circles represent patients with focal loss of aeration. Anatomical mapping of lung ultrasound reaeration score changes induced by prone positioning (lower panel). Graphical representation as a two-dimensional polar coordinate system of LUS reaeration score across the prone positioning session for each group (responders vs. not responders). Patient's response to prone positioning was defined by EELV equal or greater than $500 \mathrm{ml}$. Comparison between groups was made using a Mann-Whitney test. Significant difference correspond to a $p$ value $<0.05$. LUS lung ultrasound, $P$ P prone position

\section{Author details}

${ }^{1}$ Critical Care Unit, University Hospital of Purpan, 31300 Toulouse, France.

${ }^{2}$ ToNIC Lab, UMR UPS/INSERM 1214, Toulouse, France. ${ }^{3}$ Critical Care Unit, Narbonne Hospital, Narbonne, France.

\section{Acknowledgements}

Members of the PLUS study group (Monitoring Prone positioning lung inflation by Lung UltraSound): Amazigh Aguersif, Sihem Bouharoua, Hélène Vinour, Edith Hourcastagnou, Guillaume Ducos, Muriel Picard, Veronique Ramonda, Jean Ruiz.

\section{Compliance with ethical standards}

\section{Conflicts of interest}

The authors declare that they have no conflict of interest.

\section{Open Access}

This article is licensed under a Creative Commons Attribution-NonCommercial 4.0 International License, which permits any non-commercial use, sharing, adaptation, distribution and reproduction in any medium or format, as long as you give appropriate credit to the original author(s) and the source, provide a link to the Creative Commons licence, and indicate if changes were made. The images or other third party material in this article are included in the article's Creative Commons licence, unless indicated otherwise in a credit line to the material. If material is not included in the article's Creative Commons licence and your intended use is not permitted by statutory regulation or exceeds the permitted use, you will need to obtain permission directly from the copyright holder. To view a copy of this licence, visit http://creativecommons.org/licen ses/by-nc/4.0/.

\section{Publisher's Note}

Springer Nature remains neutral with regard to jurisdictional claims in published maps and institutional affiliations.
Accepted: 4 January 2021

Published online: 22 February 2021

\section{References}

1. Thompson BT, Chambers RC, Liu KD (2017) Acute respiratory distress syndrome. N Engl J Med 377:562-572

2. Dellamonica J, Lerolle N, Sargentini C, Beduneau G, Di Marco F, Mercat A, Richard JC, Diehl JL, Mancebo J, Rouby JJ, Lu Q, Bernardin G, Brochard L (2011) Accuracy and precision of end-expiratory lung-volume measurements by automated nitrogen washout/washin technique in patients with acute respiratory distress syndrome. Crit Care 15:R294

3. Haddam M, Zieleskiewicz L, Perbet S, Baldovini A, Guervilly C, Arbelot C, Noel A, Vigne C, Hammad E, Antonini F, Lehingue S, Peytel E, Lu Q, Bouhemad B, Golmard JL, Langeron O, Martin C, Muller L, Rouby JJ, Constantin JM, Papazian L, Leone M, Network CAEC, AzuRea Collaborative N (2016) Lung ultrasonography for assessment of oxygenation response to prone position ventilation in ARDS. Intensive Care Med 42:1546-1556

4. Silva S, Ait Aissa D, Cocquet P, Hoarau L, Ruiz J, Ferre F, Rousset D, Mora M, Mari A, Fourcade O, Riu B, Jaber S, Bataille B (2017) Combined thoracic ultrasound assessment during a successful weaning trial predicts postextubation distress. Anesthesiology 127:666-674

5. Silva S, Biendel C, Ruiz J, Olivier M, Bataille B, Geeraerts T, Mari A, Riu B, Fourcade O, Genestal M (2013) Usefulness of cardiothoracic chest ultrasound in the management of acute respiratory failure in critical care practice. Chest 144:859-865 